\title{
Synthesis of Linear Antenna Array using Genetic Algorithm to Maximize Sidelobe Level Reduction
}

\author{
T.S.Jeyali Laseetha \\ Professor, Department of Electronics and \\ Communication Engineering \\ HolyCross Engineering College, Anna University of \\ Technology, Tirunelveli, \\ Tamil Nadu, INDIA
}

\author{
Dr. R.Sukanesh \\ Professor, Department of Electronics and \\ Communication Engineering \\ Thiagarajar College of Engineering, Madurai \\ Tamil Nadu, INDIA
}

\begin{abstract}
The Genetic algorithm optimization method is used in this paper for the synthesis of antenna array radiation pattern in adaptive beamforming. The synthesis problem in this paper discussed is to finding the weights of the antenna array elements that are optimum to provide the radiation pattern with maximum reduction in the sidelobe level. This technique proved its effectiveness in improving the performance of the antenna array.
\end{abstract}

\section{Keywords}

Adaptive Beamforming, Sidelobe level, Genetic Algorithm, Linear antenna array, Pattern synthesis, convergence, Array factor.

\section{INTRODUCTION}

Adaptive beamforming is a signal processing technique in which the electronically steerable antenna arrays are used to obtain maximum directivity towards signal of interest (SOI) and null formation towards signal of not interest (SNOI) i.e instead of a single antenna the antenna array can provide improved performance virtually in wireless communication. The characteristics of the antenna array can be controlled by the geometry of the element and array excitation. But sidelobe reduction in the radiation pattern [28],[31],[32] should be performed to avoid degradation of total power efficiency and the interference suppression [1],[9] must be done to improve the Signal to noise plus interference ratio (SINR). Sidelobe reduction and interference suppression can be obtained using the following techniques: 1) amplitude only control 2) phase only control 3) position only control and 4) complex weights (both amplitude and phase control). In this, complex weights technique is the most efficient technique because it has greater degrees of freedom for the solution space. On the other hand it is the most expensive to implement in practice.

Pattern synthesis is the process of choosing the antenna parameters to obtain desired radiation characteristics, such as the specific position of the nulls [34], the desired sidelobe level [3] and beam width of antenna pattern. In literature there are many works concerned with the synthesis of antenna array. It has a wide range of study from analytical method to numerical method and to optimization methods. Analytical studies by Stone who proposed binominal distribution, Dolph the DolphChebyshev amplitude distribution, Taylor, Elliot, Villeneuve Hansen and Woodyard, Bayliss laid the strong foundation on antenna array synthesis[22]-[27]. Iterative Numerical methods became popular in 1970 s to shape the mainbeam. Today a lot of research on antenna array [1] - [12],[33] is being carried out using various optimization techniques to solve electromagnetic problems due to their robustness and easy adaptivity. One among them is Genetic algorithm [12] . R.L.Haupt has done much research on electromagnetics and antenna arrays using Genetic Algorithm [13]-[21].

In this paper, it is assumed that the array is uniform, where all the antenna elements are identical and equally spaced. The design criterion here considered is to minimize the sidelobe level [7] at a fixed main beamwidth. Hence the synthesis problem is, finding the weights that are optimum to provide the radiation pattern with maximum reduction in the sidelobe level.

\section{GENETIC ALGORITHM - AN OVERVIEW}

Genetic algorithm is a class of Evolutionary Algorithm via natural selection that works on the principle of survival of the fittest [12],[29],[30]. This optimization Algorithm is more powerful for problems with more number of variables and local minima. GA is very efficient in exploring the entire search space or the solution space, which is large and complex.

The Genetic algorithm is implemented using computer simulation, employing a population of individuals, which is the solution space. The individuals undergo the selection process by evaluating the fitness function, using operators such as mutation and crossover. Simple Genetic Algorithm was first invented by John Holland in the 1960s which provide an alternative method to solving problems, finding optimal parameters, which would otherwise be difficult for traditional methods. The selection process is important as it assures survival of the best fit individuals. The best fit individuals have the smallest cost function. The new pool of parent population is selected from among the elite of the parents and children.

In Genetic Algorithm reproductive cycle may be represented as shown below in Fig 1. 


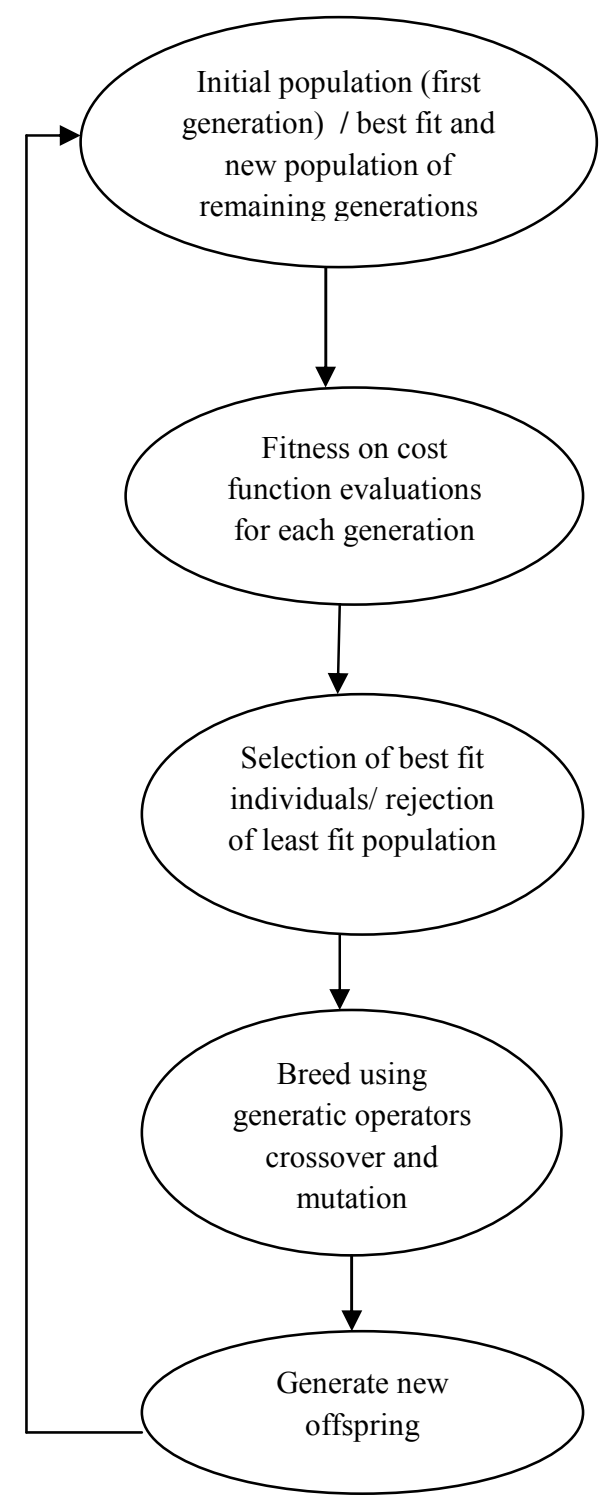

Fig 1: GA reproduction cycle

The important parameters of GA can be summarized viz.,

1. Crossover type and crossover rate.

2. Mutation type and mutation rate.

3. Population size.

4. Selection procedure.

5. Number of generations.

They are defined as given below

- Crossover - this is an exchange of substrings denoting chromosomes, for an optimization problem. It may be a single point cross over, two point cross over, cut and splice, uniform crossover or half uniform crossover

- Mutation - the modification of bit strings in a single individual
- Population - the number of chromosomes considered in one generation

- Selection - evaluation of the fitness criterion to choose which individuals from a population will go on to reproduce. Some general methods used are Roulette Wheel Selection and Tournament Selection

- Number of generations - the maximum number of generations that the genetic algorithm can evolve into, before terminating.

The cycle is repeated until a termination condition has been reached such as

1. A solution that satisfies the minimum criteria.

2. Reaching the specified number of generations.

3. Reaching the specified Computation time.

4. Arriving fitness value and

5. Manual inspection.

Traditionally, solutions are represented using fixed length strings, especially binary strings, but alternative encodings have been developed.

Current GA theory consists of two main approaches - Markov chain analysis and schema theory. Markov chain analysis is one which utilizes the stochastic dynamics of a GA system. But its dynamics are difficult to describe mathematically. A schema is a conceptual system for understanding knowledge and how it is used. According to this theory, schemata represent knowledge about concepts: objects and the relationships they have with other objects, situations, events, sequences of events, actions, and sequences of actions.

\section{UNIFORM LINEAR ANTENNA}

\section{ARRAY}

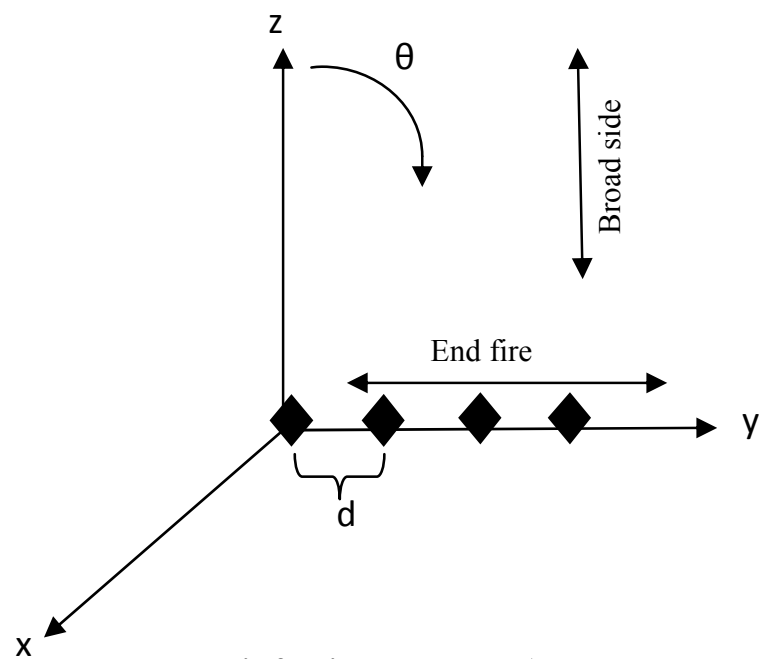

Fig 2: Linear antenna Array 
In linear antenna array, all the antenna elements are arranged in a single line with equal spacing between them. Due to far field consideration, the incident wave is assumed to be a plane wave which causes a linear gradient time delay between the antenna elements that is proportional to the angle of incidence. This time delay will lead to progressive phase shift between the elements. In Fig 2 it is shown that the antenna elements are arranged with uniformly spacing, in a straight line along the $\mathrm{y}$-axis, and $\mathrm{N}$ is the total number of elements in the antenna array with the physical separation distance as $d$, and the wave number of the carrier signal is $k=2 \pi / \lambda$. When $k d$ is equal to $\pi$ (or $d=\lambda / 2$ )

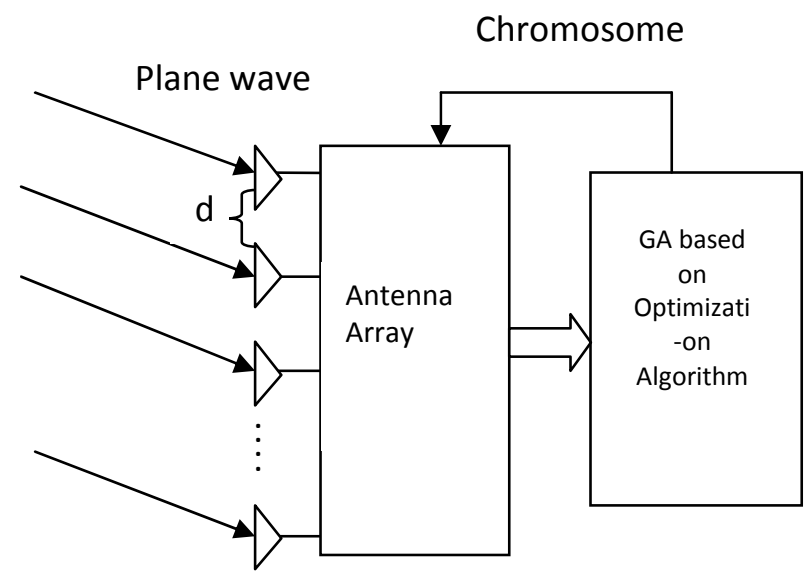

Fig 3: Antenna Array

the antenna array has maximum gain with no grating lobes. The phase shift between the elements experienced by the plane wave is $k d \cos \theta$. Weights can be applied to the individual antenna signals before the array factor (AF) is formed to control the direction of the main beam as shown in Fig 3. This corresponds to a multiple-input-single-output (MISO) system. The total AF is just the sum of the individual signals, given by [9]

$$
A F=\left|\left(\sum_{n=1}^{N} E_{n}\right)\right|=\sum_{n=1}^{N} e^{j K_{n}}
$$

The factor $K=\left(n * k d \cos \theta+\beta_{n}\right)$ is the phase difference. Final simplification of equation (1) is by conversion to phasor notation. Only the magnitude of the AF in any direction is important, as the absolute phase has no bearing on the transmitted or received signal. Therefore, only the relative phases of the individual antenna signals are important in calculating the AF.

\section{PROBLEM FORMULATION}

Consider an array of antenna consisting of $\mathrm{N}$ number of elements. It is assumed that the antenna elements are symmetric about the center of the linear array. The far field array factor of this array with an even number of isotropic elements $(2 \mathrm{~N})$ can be expressed as

$$
A F(\theta)=2 \sum_{n=1}^{N} a_{n} \cos \left(a \frac{\pi}{\lambda} d_{n} \sin \theta\right)
$$

where $\mathrm{a}_{\mathrm{n}}$ is the amplitude of the $\mathrm{n}^{\text {th }}$ element, $\theta$ is the angle from broadside and $d_{n}$ is the distance between position of the $n^{\text {th }}$ element and the array center. The main objective of this work is to find an appropriate set of required element amplitude $a_{n}$ that achieves interference suppression with maximum sidebobe level reduction.

To find a set of values which produces the array pattern, the algorithm is used to minimize the following cost function

$$
c f=\sum_{\theta=-90^{\circ}}^{90^{\circ}} W(\theta)\left[F o(\theta)-F_{d}(\theta)\right]
$$

where $F o(\theta)$ is the pattern obtained using our algorithm and $F_{d}(\theta)$ is the pattern desired. Here it is taken to be the Chebychev pattern with SLL of $-13 \mathrm{~dB}$ and $W(\theta)$ is the weight vector to control the sidelobe level in the cost function. The value of cost function is to be selected based on experience and knowledge.

\section{RESULTS AND DISCUSSION}

The antenna model consists of 20 elements and equally spaced with $\mathrm{d}=0.5 \lambda$ along the $\mathrm{y}$-axis. Voltage sources are at the center segment of each element and the amplitude of the voltage level is the antenna element weight. Only the voltage applied to the element is changed to find the optimum amplitude distribution, while the array geometry and elements remain constant. A continuous GA with a population size 10 and a mutation rate of 0.35 is run for a total of 500 generations using MATLAB and the best result was found for each iteration. The cost function is the minimum sidelobe level for the antenna pattern. Fig 4 shows that the antenna array with $\mathrm{N}=8$ elements has been normalized for a gain of $0 \mathrm{~dB}$ along the angle $0^{\circ}$ and the maximum relative side lobe level of $-15 \mathrm{~dB}$.

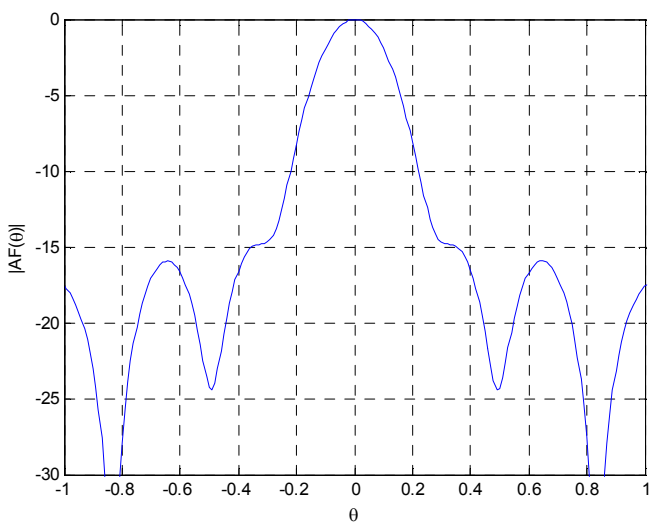

Fig 4: Optimized Radiation pattern with reduced sidelobe level of $-15 \mathrm{~dB}$ for $\mathrm{N}=8$ elements 


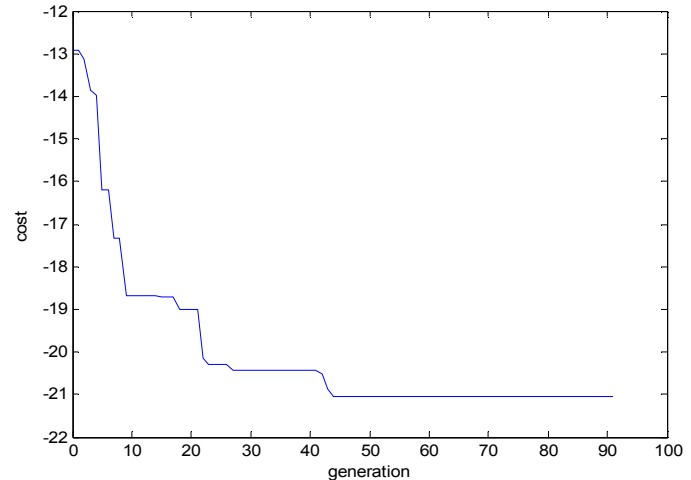

Fig 5: Convergence of sidelobe level with respect to evolving generations for $\mathbf{N}=8$ elements.

Fig 5 shows the convergence of the algorithm for maximum reduction in the relative sidelobe level with $\mathrm{N}=8$ elements. It starts from $-13 \mathrm{~dB}$ which is the optimized value of chebychev pattern for the RSLL and after 8 iterations it reaches $-18.8 \mathrm{~dB}$ and after 43 generations it converges to a maximum reduction of $-21 \mathrm{~dB}$. Fig 6 shows the optimized radiation pattern with relative sidelobe level of $-15 \mathrm{~dB}$ with $\mathrm{N}=16$ and Fig 8 shows its convergence curve. The convergence curve shows that it converges to

$-19.3 \mathrm{~dB}$ after 54 generations. Changing the number of elements causes the contiguous GA to get different optimum weights. Among $\mathrm{N}=8,16,20$, and $24, \mathrm{~N}=20$ performed well and thus selected as optimized element number. The corresponding array pattern for $\mathrm{N}=8,16,20$, and 24 are shown in Fig 7. In this the radiation pattern for $\mathrm{N}=20$ has the best directivity with minimum relative sidelobe level of $-14.67 \mathrm{~dB}$ below the main beam. Fig 8 and Fig 9 show the convergence of sidelobe level for $\mathrm{N}=16$ and 20 respectively. Fig 10 and 12 show the optimized radiation pattern with relative sidelobe level of $-18.7 \mathrm{~dB}$ for $\mathrm{N}=20$ and rsll of $-14.97 \mathrm{~dB}$ for $\mathrm{N}=24$ elements respectively. Fig 11 shows the convergence curve for $\mathrm{N}=24$ elements.

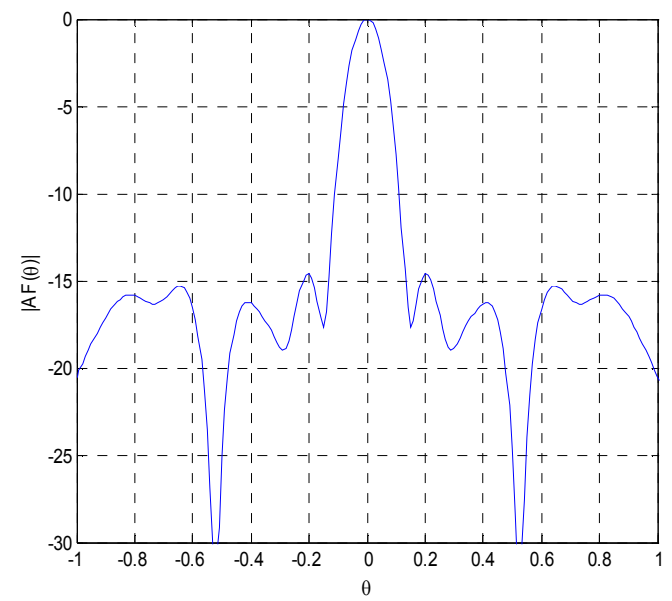

Fig 6: Optimized Radiation pattern with reduced sidelobe level of $-15 \mathrm{~dB}$ for $\mathrm{N}=16$ elements

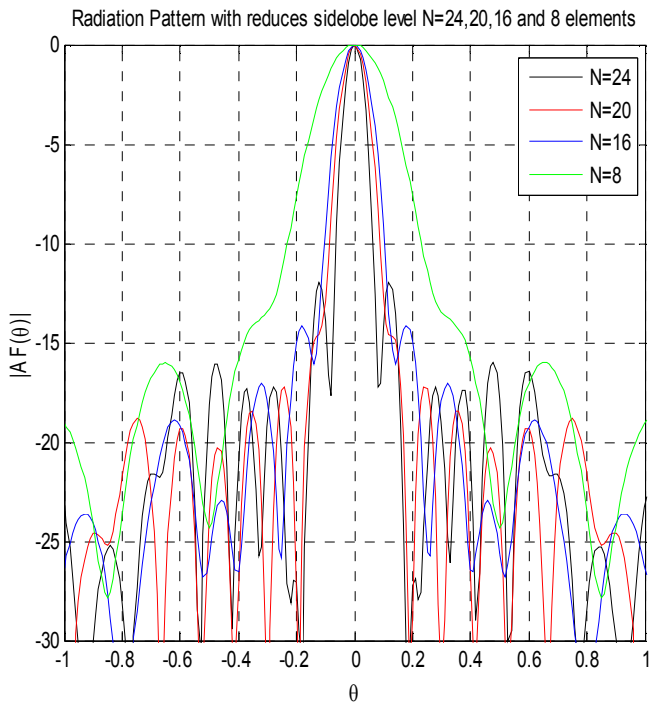

Fig 7 : The optimized radiation pattern with reduced sidelobe level for $\mathrm{N}=8,16,20$, and 24

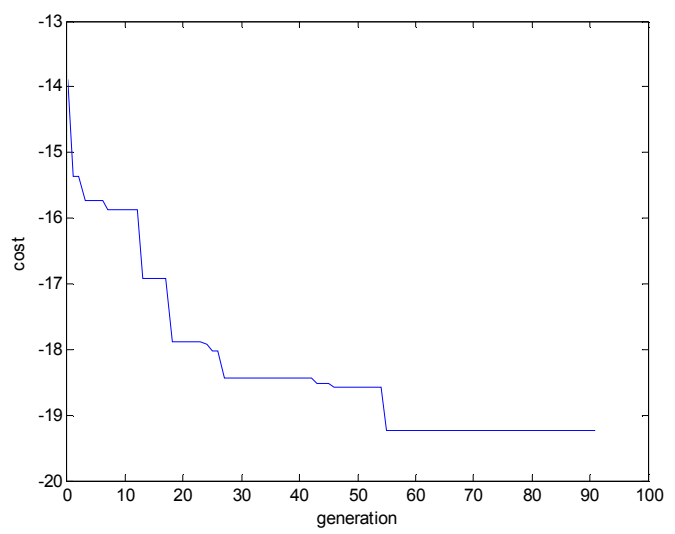

Fig 8: Convergence of sidelobe level with respect to evolving generations for $\mathrm{N}=16$ elements

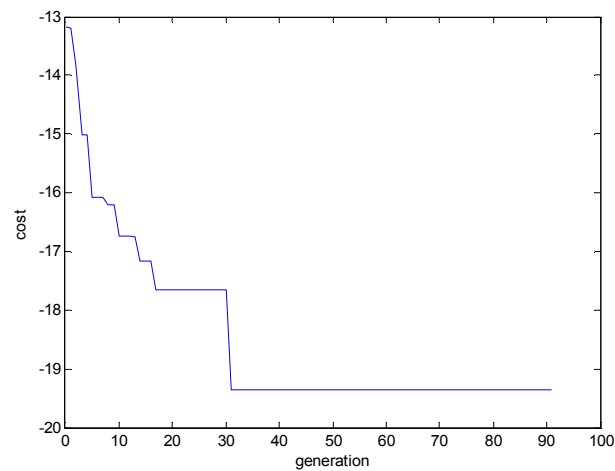

Fig 9: Convergence of sidelobe level with respect to evolving generations for $\mathbf{N}=\mathbf{2 0}$ elements 


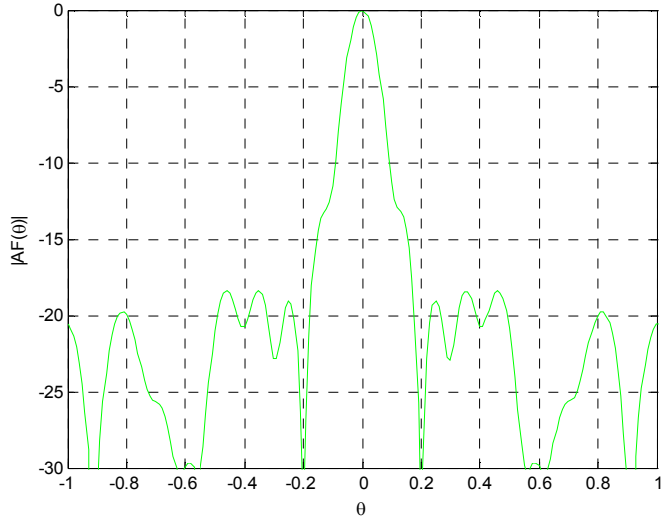

Fig 10: The optimized radiation pattern with reduced sidelobe level for number of elements $N=\mathbf{2 0}$

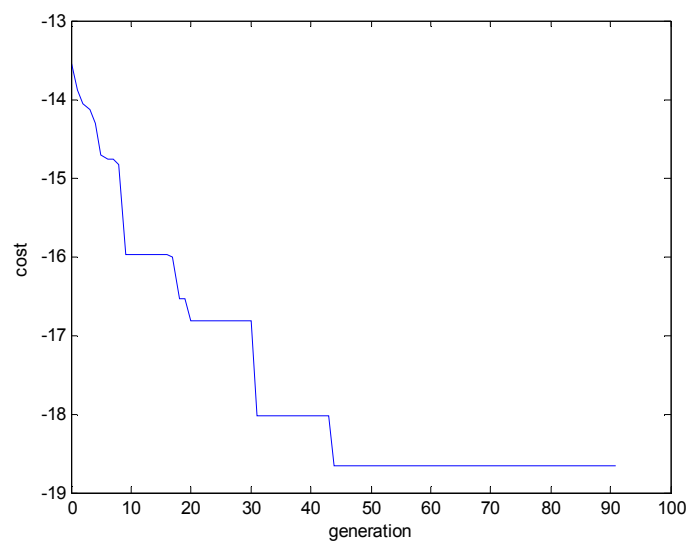

Fig 11: Convergence of sidelobe level with respect to evolving generations for $\mathrm{N}=24$ elements.

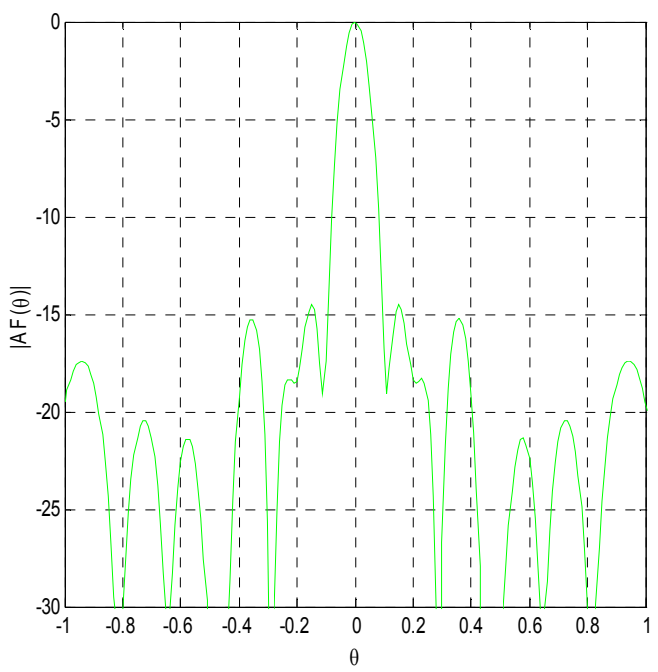

Fig 12: The optimized radiation pattern with reduced sidelobe level for number of elements $N=\mathbf{2 4}$
The obtained costs are ranked from best to worst. The most among suitability criteria is to discard the bottom half and to keep the top half of the list. But in our program the selection criteria is to discard any chromosome that has relative sidelobe level less than $-15 \mathrm{~dB}$. Table 2 shows the cost function relative to the population that has a SLL less than $-15 \mathrm{~dB}$. Among 10 populations only 5 are selected. This limitation speeds up the convergence of the algorithm. After this natural selection the chromosomes mate to produce offsprings. Mating takes place by pairing the surviving chromosome. Once paired, the offspring consists of genetic material from both parents

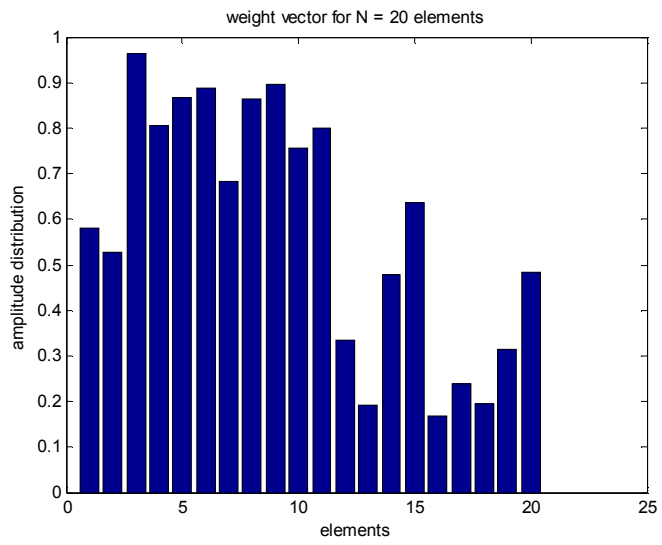

Fig 13: Amplitude distribution for optimized antenna array with $\mathbf{N}=\mathbf{2 0}$ elements

Fig 13 shows the amplitude excitation for optimized antenna array as given in Table 1 .

The Genetic algorithm has many variables to control and tradeoffs to consider.

1) Number of Chromosomes and initial random Population, more number of chromosomes provide better sampling number, solution space but at the cost of slow convergence.

2) Generating the random list, the type of probability distribution and weighting of the parameters has a significant impact on the convergence time.

3) Natural selection method is employed to decide which chromosome to discard.

4) Crossover the chromosome for mating, the chromosome may be paired from top to bottom randomly best to worst.

5) Mutation rate is selected to mutate a particular chromosome. Mutate does not permit the algorithm to get stuck at local minimum.

6) Stopping Criteria, set in this program are maxgen $=$ 500 , maxfun $=1000$ and $\quad$ mincost $=-50 \mathrm{~dB}$.

In this paper the Genetic Algorithm has converged well for a variant of options mentioned above with some trade offs to have main impact on convergence speed. 


\section{CONCLUSION}

In this paper Genetic algorithm is used to obtain minimum sidelobe level relative to the main beam on both sides of $0^{\circ}$. The specialty of the Genetic algorithm is that it can optimize the large number of discrete parameters. Genetic algorithm is an intellectual algorithm searches for the optimum element weight of the array antenna. This paper demonstrated the different ways to apply Genetic algorithm by varying the values of mutation, population size, number of elements to optimize the array pattern. The best obtained results are explained in the previous sections.

\section{REFERENCES}

[1] M.A.Panduro, "Design of Non-Uniform Linear Phased Arrays using Genetic Algorithms To Provide Maximum Interference Reduction Capability in a Wireless Communication System", Journal of the Chinese Institute of Engineers,Vol.29 No.7,pp 1195-1201(2006).

[2] Stephen Jon Blank, "On the Empirical optimization of Antenna Arrays", IEEE antenna and Propagation Magazine,47, 2, pp.58-67, April 2005.

[3] Aniruddha Basak.et.al, "A Modified Invasive Weed Optimization Algorithm for Time- Modulated Linear Antenna Array Synthesis", IEEE Congress on Evolutionary Computation (CEC) DOI:10.1109/CEC.2010.5586276 pp.1-8, 2010.

[4] Aritra Chowdhury et.al. "Linear Antenna Array Synthesis using Fitness-Adaptive Differential Evolution Algorithm", IEEE Congress on Evolutionary Computation (CEC) 2010 pp.1-8,DOI.2010/5586518.

[5] T.B.Chen,Y,B.Chen,Y.C.Jiao and F.S.Zhang, "Synthesis of Antenna Array Using Particle Swarm Optimization”, Asia-Pacific Conference proceedings on Microwave Conference,2005 ,APMC,2005,pp.4.

[6] Peiging Xia and Mounir Ghogho, "Evaluation of Multiple Effects Interference Cancellation in GNSS using SpaceTime based Array Processing", International Journal of Control, Automation, and Systems, vol. 6, no. 6, pp. 884893, December 2008.

[7] Aniruddha Basak, Siddharth Pal, Swagatam Das and Ajith Abraham, "Circular Antenna Array Synthesis with a Differential Invasive Weed Optimization Algorithm", 10th International Conference on Hybrid Intelligent Systems (HIS 2010), Atlanta, USA (Accepted, 2010).

[8] Oscar Quevedo-Teruel and Eva Rajo-Iglesias, "Application of Ant Colony Optimization Based Algorithm to solve Different Electromagnetic Problems", Proc.EuCAP 2006, Nice, France 6-10, November 2006.

[9] Peter J.Bevelacqua and Constantine A.Balanis, "Optimizing Antenna Array Geometry for Interference Suppression", IEEE Transaction on Antenna And Propagation, Vol.55, no.3 pp 637-641,March 2007.

[10] Stephen J.Blank, “Antenna Array Synthesis Using Derivative, Non-Derivative and Random Search Optimization", IEEE Sarnoff Symposium, DOI 10.1109/SARNOF. 2008.4520115, pp 1-4,May 2008.
[11] Korany R. Mahmoud,et.al., "Analysis of Uniform Circular Arrays for Adaptive Beamforming Application Using Particle Swarm Optimization Algorithm”, International Journal of RF and Microwave Computer-Aided Engineering DOI 101.1002 pp.42-52.

[12] David E.Goldberg, John H.Holland, "Genetic Algorithm and Machine Learning", Kluwer Academic Publishers, Machine Learning 3 Issue 2-3, pp 95-99, 1998.

[13] R.L.Haupt, "Directional Antenna System Having Sidelobe Suppression,Us Patent 4, pp571-594 Feb 18,1986 .

[14] R.L.Haupt, "Thinned arrays using genetic algorithm", IEEE Transaction on Antenna and Propagation, Vol.12 Issue 7, pp 993-999 July1994.

[15] R.L.Haupt, "Optimum quantized low sidelobe phase tapers for array”,.IEEE Electronics Lett 31(14) pp11171118 July 1995.

[16] R.L.Haupt, "Synthesizing low sidelobe quantized amplitude and phase tapers for linear arrays using genetic algorithms, Proc Inte.Conf. Electromagnetics in Advanced Application, Torino, Italy, pp 221-224 Sept.1995.

[17] R.L.Haupt, "Adaptive Nulling With Weight Constraints", Progress In Electromagnetics Research B, Vol. 26, pp 23$38,2010$.

[18] C.L.Dolph, "A current distribution for broadside arrays which optimizes the relationship between beam width and side-lobe level," Proc IRE 34 pp.3335-348 June 1946.

[19] T.T Taylor, "Design of line source antennas for narrow beamwidth and low side lobes", IRE AP Trans 4 pp 1628 Jan 1955.

[20] R.S.Elliott, “Antenna Theory and Design”, Prentice-Hall, New York 1981.

[21] A.T.Villeneuve, Taylor, "Patterns for discrete pattern arrays", IEEE AP- S Trans 32(10) pp 1089-1094 October 1984.

[22] W.W.Hansen and J.R.Woodyard, "A new principle in directional antenna design”, Proc. IRE 26 pp 333-345 March 1938.

[23] E.T.Bayliss, "Design of Monopulse Antenna difference Patterns with low sidelobes", Bell Syst. Tech.J.47 pp623650 May-June 1968.

[24] W.L.Stutzman and E.L Coffey, "Radiation pattern synthesis of planar antennas using the iterative sampling method", IEEE Trans.AP 23(6) pp762-769 November 1975.

[25] J.H.Holland, "Adaptation in Natural and Artificial Systems, Univ. Michigan Press,Ann Arbor 1975.

[26] D.E.Goldberg, "Genetic Algorithm in search optimization and Machine Learning Addison-Wesley, New York, 1989.

[27] B.Widrow et.al. “Adaptive antenna system”, IEEE.Proc 55(12) pp2143-2159 Dec 1967. 
[28] R.A.Monzingo and T.W.Miller, "Introduction to Adaptive Arrays", SciTech Publishing, Rayleigh NC 2003.

[29] Y.Lu and B.K Yeo, "Adaptive wide null steering for digital beamforming array with complex coded genetic algorithm", Proc.IEEE Phased Array System and Technology Symp pp 557-560 May 2000.

[30] R.L.Haupt, “An introduction to genetic algorithm for electromagnetics”, IEEE Antenna. Propagation Mag 37(2) , pp7-15 April 1995.
[31] R.L.Haupt, "Generating a plane wave in the near field with a planar array antenna Micrw.J.46 (9) pp 152-158 Aug 2003.

[32] R.L.Haupt and Sue Ellen Haupt, "Practical Genetic Algorithm", $2^{\text {nd }}$ ed., Wiley, New York,2004.

[33] R.L.Haupt, Douglas H.Werner, "Genetic Algorithm in Electomagnetics”, Wiley interscience Publication 2007.

Table 1: Amplitude excitation values for $\mathrm{N}=20$ elements corresponding to Fig no.13

\begin{tabular}{|c|c|}
\hline $\mathbf{W}_{\mathbf{n}}$ & $\begin{array}{c}\text { Amplitude } \\
\text { excitation }\end{array}$ \\
\hline $\mathrm{W}_{1}$ & 0.9028 \\
$\mathrm{~W}_{2}$ & 0.9645 \\
$\mathrm{~W}_{3}$ & 0.7259 \\
$\mathrm{~W}_{4}$ & 0.6910 \\
$\mathrm{~W}_{5}$ & 0.7026 \\
$\mathrm{~W}_{6}$ & 0.9491 \\
$\mathrm{~W}_{7}$ & 0.7789 \\
$\mathrm{~W}_{8}$ & 0.3478 \\
$\mathrm{~W}_{9}$ & 0.5097 \\
$\mathrm{~W}_{10}$ & 0.6319 \\
$\mathrm{~W}_{11}$ & 0.5358 \\
$\mathrm{~W}_{12}$ & 0.5625 \\
$\mathrm{~W}_{13}$ & 0.4696 \\
$\mathrm{~W}_{14}$ & 0.4828 \\
$\mathrm{~W}_{15}$ & 0.2411 \\
$\mathrm{~W}_{16}$ & 0.5464 \\
$\mathrm{~W}_{17}$ & 0.0060 \\
$\mathrm{~W}_{18}$ & 0.1060 \\
$\mathrm{~W}_{19}$ & 0.4043 \\
$\mathrm{~W}_{20}$ & 0.4334 \\
& \\
\hline
\end{tabular}

Table 2: Population and Respective Cost Function Values

\begin{tabular}{|c|l|l|l|l|l|l|l|l|l|}
\hline Index & \multicolumn{70}{|c|}{ Chromosome (weight vector) } & $\begin{array}{c}\text { Relative } \\
\text { sidelobe } \\
\text { level (dB) }\end{array}$ \\
\hline 1 & 0.8933 & 0.9059 & 0.7956 & 0.7167 & 0.5880 & 0.3725 & 0.2648 & 0.2232 & -26.9682 \\
\hline 2 & 0.8933 & 0.7659 & 0.5982 & 0.5391 & 0.9296 & 0.7216 & 0.7412 & 0.2059 & -19.0861 \\
\hline 4 & 0.4635 & 0.7659 & 0.6338 & 0.5391 & 0.9296 & 0.2594 & 0.7412 & 0.2059 & -18.2047 \\
\hline 7 & 0.8933 & 0.7659 & 0.8712 & 0.4302 & 0.9296 & 0.2594 & 0.7412 & 0.2059 & -17.5515 \\
\hline 5 & 0.8374 & 0.4270 & 0.7824 & 0.5322 & 0.5425 & 0.8238 & 0.9641 & 0.3051 & -17.5173 \\
\hline
\end{tabular}

